\title{
Do current statistical learning capture stable individual differences in children? An investigation of task reliability across modalities
}

\section{Inbal Arnon (Inbal.arnon@mail.huji.ac.il) 1}

${ }^{1}$ Department of Psychology, Hebrew University, Jerusalem, Israel

\section{Highlights}

- Growing evidence for positive correlations between SL performance and language outcomes in development.

- Findings based on a small set of tasks developed for assessing group-level performance that may not be suited to measure individual differences

- No study to date has examined the reliability of these tasks in children, a crucial prerequisite for using them to assess individual differences

- We assess the reliability of three SL tasks across modalities (visual, linguistic-auditory, non-linguistic auditory) and the digit span in children (mean age 8;2) and adults.

- Tasks show reliability in adults, but not in children, raising concern about their use to predict and explain individual differences in development.

\begin{abstract}
Do commonly used statistical learning measures capture stable individual differences in children? Infants, children and adults are capable of using statistical learning to extract information about their environment. While most studies look at group-level performance, a growing literature examines individual differences in SL and their relation to language learning outcomes: individuals who are better at SL are expected to show better linguistic abilities. Accordingly, studies have found positive correlations between SL performance and
\end{abstract}




\section{Under review}

language outcomes in both children and adults. However, these studies often use tasks designed to explore group-level performance without modification, resulting in psychometric shortcomings (Siegelman et al. 2017a). In particular, they use relatively few testing trials, repeat items during testing, and assess learning using forced choice trials, all of which impact task reliability in adults (Siegelman et al. 2017b). Even though similar measures are used to assess individual differences in children, no study to date has examined their reliability in development. We examine the reliability of common SL measures in children, and ask if they are similarly reliable in children and adults. We assess the reliability of three SL tasks (two auditory and one visual) in children (mean age 8;2) and adults two months apart. While the tasks showed moderate reliability in adults, they did not capture stable individual variation in children where test-retest and split-half reliability fell well below psychometric standards. These findings raise significant concerns about the use of current SL measures to predict and explain individual differences in development.

Keywords: statistical learning; individual differences; reliability; domain-generality; children 


\section{Introduction}

Infants, children and adults are constantly exposed to recurring patterns in their environment, and manage to learn and generalize from them. This ability - often called statistical learning (SL) - is postulated to be one of the important mechanisms in language learning and learning more generally (e.g., Erickson \& Thiessen, 2015). Statistical learning in infants was demonstrated in a seminal study showing that 8-month-old infants can use distributional information about syllable co-occurrence to discover word boundaries (Saffran, Aslin, \& Newport, 1996). This study led to a surge in studies probing this ability in both infants and adults. Research over the past 20 years has shown that statistical learning is present from early infancy (Bulf, Johnson, \& Valenza, 2011), is found across modalities (visual, auditory, tactile, Conway \& Christiansen, 2005; Kirkham et al., 2002) and can be used to learn a range of linguistic properties (phonetic categories, word order, phrase structure, see Romberg \& Saffran, 2010) for a review). These studies, which assess learning at the group level, serve as a 'proof of concept': they show that humans are capable of using distributional information to extract complex structure from their environment.

Recent years have seen growing interest in the predictive power of SL as a way to assess and predict individual differences in language learning (Siegelman, Bogaerts, \& Frost, 2017; Siegelman \& Frost, 2015). If SL plays an important part in language acquisition, we would expect it to be correlated with various language outcomes such that individuals who are better at SL will also show better linguistic abilities. Similarly, difficulty with SL may contribute to impaired language performance in children with a-typical language development (Mainela-Arnold \& Evans, 2014). Indeed, a growing number of studies find correlations between SL measures and language outcomes. In adults, performance on visual SL has been related to better literacy outcomes in L2 (Frost, Siegelman, Narkiss, \& Afek, 2013), syntactic processing (Misyak \& Christiansen, 2012; Misyak, Christiansen, \& Tomblin, 2010), and 


\section{Under review}

speech perception (Conway, Bauernschmidt, Huang, \& Pisoni, 2010). Similar findings have been reported for children where visual SL is predictive of syntactic processing (Kidd, 2012; Kidd \& Arciuli, 2016), early literacy skills (Arciuli \& Simpson, 2012), and vocabulary size (Spencer, Kaschak, Jones, \& Lonigan, 2015). Work with infants also suggests links between SL performance and later learning with visual SL predicting later vocabulary size (Ellis, Robeldo Gonzales \& Doek, 2013; Shafto, Conway, Field, \& Houston, 2012) and real-time processing (Lany, Shoaib, Thompson, \& Graf Estes, 2017).

Taken together, these findings suggest a strong link between SL performance and variations in language outcomes, compatible with the postulated role of SL in language acquisition. However, this interpretation is dependent on the reliability of the SL measures used: the correlations between SL and other measures can be meaningfully interpreted only if SL measures are reliable. Otherwise we cannot know how much of the observed interindividual variation reflects measurement error and how much reflects meaningful variation. Despite extensive use in the past 20 years, relatively little work has examined the reliability of SL measures: such investigations were less pressing when SL tasks were used to assess group-level performance. However, as their use as measures of individual differences gained prominence, so did the need to examine and validate their psychometric properties.

A series of recent studies has raised questions about the suitability of commonly used SL tasks for assessing individual differences in adults (Siegelman, Bogaerts, Christiansen \& Frost, 2017; Siegelman, Bogaerts \& Frost, 2017). These studies highlight the fact that many studies of individual differences of SL have tended to adopt the same tasks used to explore group-level performance without modifying them, resulting in psychometric shortcomings. Many adult studies employ a word segmentation task modeled on the original Saffran et al. (1996) study (often called ASL) or a visual parallel that uses shapes instead of syllables (VSL, modeled on Turk-Browne, 2005). In these tasks, participants have to detect recurring triplets 


\section{Under review}

(visual or auditory) in a continuous stream based on their statistical properties (see Table 1 in Siegelman et al 2017a for a summary of methods used in recent studies). While the implementation of these tasks differs across studies, they share several properties that undermine their suitability as a measure of individual differences (Siegelman et al. 2017; Siegelman, Bogaerts \& Frost, 2017). In particular, they have relatively few testing trials, all at the same level of difficulty; they repeat items during testing; they use two-alternative forced choice trials to assess learning after exposure; and show accuracy that is usually not much higher than chance, meaning that many participants perform at chance level. These characteristics reduce task reliability and may lead to the detection of spurious correlations on the one hand, and the under-detection of true correlations on the other (see Siegelman, Bogaerts \& Frost 2017 for simulations demonstrating this). This literature outlines several ways that SL measures can be improved, for example by increasing the number and type of test trials (Siegelman et al. 2017), making the testing more implicit (Isbilen, McCauley, \& Christiansen, 2017), or using online measures of learning during exposure (Siegelman, Bogaerts, Kronenfeld \& Frost, 2017), all of which increase reliability. Despite these shortcomings, commonly used SL measures (the ASL and VSL discussed above) seem to capture stable individual variation in adults: They show test re-test reliability between sessions and internal consistency within sessions, though these measures are often below psychometric standards (test re-test of around 0.5: Siegelman \& Frost, 2015, Potter, Wang, \& Saffran, 2017; split-half reliability of around 0.8, Siegelman, Bogaerts \& Frost, 2017).

Even though very similar measures are used to assess individual differences in children, no study to date has examined their reliability in development: we do not know is these measures capture stable variation in children ${ }^{1}$. Such an investigation is necessary to

\footnotetext{
1 The tasks used with infants have similar properties but differ in that learning is assessed implicitly, a point which we return to in the discussion. We focus here on tasks that assess learning in the same way in children and adults.
} 


\section{Under review}

interpret and validate the reported correlations between SL and language outcomes. Moreover, there are several reasons to suspect that children will be more affected by the shortcomings of these tasks. First, the use of relatively few test trials and the repetition of items and foils during testing may have a larger effect on children, who have smaller attention and memory resources (e.g., Gathercole et al. 1994; Plude et al. 1994). Second, the use of explicit judgments may introduce additional noise into children's performance, especially when they are asked to compare the same form repeatedly (see recent debate in the theory of mind literature on the difference between online and offline measures of false belief, e.g., Southgate, V., Senju, A., \& Csibra, G., 2007). Third, children's generally lower accuracy on these tasks means that even more of them are not showing above chance performance, further undermining their use for predicting variations in language outcomes. Finally, the range of SL tasks in the child individual difference literature is even more limited than in the adult literature: four of the five published studies with children use either the ASL or the VSL with similar exposure and testing properties (4-6 triplets; 36-64 testing trials; learning assessing using 2AFC-forced choice trials with repetition of test items and foils during testing). That is, our knowledge about the relation between SL and language outcomes in children is based on findings from a small set of tasks that are not ideally suited to measure individual differences.

In line with these concerns, the correlations between SL and language outcomes during development are small, and weaker than those found for adults. While the correlations in adults tend to be moderate $(r=0.4-0.6)$, those of children are lower, suggesting a weaker relationship between the variance captured by SL measures and language outcomes (ranging between $r=.1$ and .34 , see Table 1 in Siegelman, Bogaerts, Christiansen, \& Frost, 2017). Moreover, the pattern of correlations is not consistent even when using the same language measures. For instance, Kidd (2012) and Kidd \& Arciuli (2016) found no correlation between SL and vocabulary measures in children while Spencer et al. (2015) did. The low correlations 


\section{Under review}

and the fluctuations between studies may both stem from the tasks not having sufficient reliability.

In the current study, we examine the reliability of SL measures in children, and ask if they are similarly reliable in children and adults. We do so by assessing the reliability of three SL tasks (two auditory and one visual) that are closely modeled on ones used in the child individual difference literature. The first experiment looks at adults with the aim of providing a reliability baseline for the precise measures we then test with children: it is theoretically possible that child-friendly tasks have low reliability also in adults because they often have shorter exposure times and a reduced number of test items. The second experiment examines the reliability of the same three tasks in children aged seven to nine years (mean age $8 ; 2$ ). We evaluate reliability in two ways: (1) by looking at the internal reliability and consistency of tasks within each session (using split-half reliability and Cronbach alpha coefficients) and (2) by examining their test re-test reliability two months apart. We also assessed verbal working memory using the digit span task in both sessions. Collecting this measure serves as a sanity check: it is known to be stable within individual children and is therefore expected to show high test re-test reliability (Gathercole, Willis, Baddeley, \& Emslie, 1994). We focus on children at ages seven to nine because children in this age group showed group-level learning on the same SL tasks (Raviv \& Arnon, 2017). If SL tasks tap onto stable individual differences in children, they should be correlated across the two test sessions. If they do not, this would raise significant concerns about their use to predict and explain individual differences in development.

The SL tasks were closely modeled on ones previously used in child individual difference research (e.g., (Kidd, 2012; Kidd \& Arciuli, 2016; Spencer et al. 2015). We used (1) a linguistic auditory task study where participants are exposed to recurring triplets of syllables, (2) a non-linguistic auditory task where the syllables were replaced with familiar 


\section{Under review}

sounds (dog barking, bell, drum), using stimuli from Siegelman, Bogaerts, Arciuli \& Frost (under review), and (3) a visual task where participants saw recurring triplets of familiar object drawings (e.g., car, door, plate). This task resembled the child-friendly visual SL task developed by (Arciuli \& Simpson, 2011). All three tasks require learners to detect recurring triplets in a continuous temporal input. While the tasks differed in the stimuli used (syllables vs. non-linguistic sounds vs. drawings), they were comparable in terms of the distributional information learners were exposed to, the number of repetitions of each triplet, and the number and nature of test trials.

\section{General Method}

\section{Materials}

Since we used the same SL tasks with children and adults we describe them here and then report the results of the two studies. In all three SL tasks participants were exposed to a continuous stream made up of five recurring triplets. The transitional probabilities (TPs) between elements within a triplet were always 1 , while the TPs between triplets were 0.25 (because stimuli were not repeated across triplets and because each triplet could be followed by any of the other four). Following exposure, participants' knowledge of the triplets was assessed using 25 two-alternative forced-choice trials (2AFC). We describe the stimuli for each task separately and then describe the identical testing phase (see Appendix A for the full stimuli list). Verbal working memory was assessed using the digit span task where participants have to recall lists of numbers growing in size (Kaufman, 1994). With children, we only used the digits one to five to prevent arithmetic ability from affecting performance (Havron \& Arnon, 2017).

Linguistic Auditory task. The auditory stimuli consisted of a synthesized "alien" language, containing five unique tri-syllabic words, made up of 15 different syllables synthesized using 


\section{Under review}

PRAAT (Boersma, 2001). All words were then synthesized using the PRAAT software in order to control for syllable duration and frequency. Average word length was $850 \mathrm{~ms}$. Words were concatenated together in a semi-randomized order (with the constraint that no word would appear twice in a row) to create an auditory familiarization stream. The exposure phase lasted 2;20 minutes, with each word repeated 32 times with no breaks between words and no prosodic or co-articulation cues in the stream to indicate word boundaries. In this task only, the familiarization stream was identical for all participants: all participants heard the same triplets in both sessions. Using the same familiarization stream between participants is a common feature of ASL tasks (starting from Saffran et al. 1996 and repeated across many studies). We used the same stream in both sessions because group performance on this task seems to be affected by the exact syllable combinations in ways that are not fully understood (Erickson, Kaschak, Thiessen \& Berry, 2015; Siegelman et al., under review). Our concern was that changing the triplets would introduce variation we cannot predict (we return to the possible implications of this in the discussion).

Non-linguistic Auditory task. This task had very similar properties to the linguistic task except that syllables were replaced with familiar non-linguistic sounds (e.g., bell, dog barking). The auditory stimuli contained five unique triplets made up of 15 different sounds. Unlike the linguistic task, triplets were generated anew for each participant, so that each participant heard a different set of triplets. Triplets were changed between sessions so that participants did not hear the same triplets in both. Average sound length was $500 \mathrm{~ms}$. Triplets were concatenated in a semi-randomized order, with the constraint that no triplet would appear twice in a row. The exposure phase lasted 3:00 minutes, with each triplet repeated 24 times.

Visual task. This task had similar properties to the previous two but in the visual domain. The visual stimuli consisted of a continuous temporal stream of black and white drawing of familiar objects (e.g., plane, door), containing five unique triplets of drawings (a 


\section{Under review}

total of 15 different drawings). We selected line drawings from the Snodgrass (1980) database that have high naming agreement based on Alario and Ferrand (1999). All names had high frequency in Hebrew and early Age-of-Acquisition (Maital, Dromi, Sagi, \& Bornstein, 2000). Each drawing appeared on the screen for $500 \mathrm{~ms}$, with a $100 \mathrm{~ms}$ break between figuresresulting in a $1800 \mathrm{~ms}$ presentation time for each triplet. The triplets were generated anew for each participant and changed between sessions. For each participant, the five triplets were concatenated together in a semi-randomized order (with the constraint that no triplet would appear twice in a row). The exposure phase lasted 3:30 minutes, with each triplet repeated 24 times.

The test phase. The test phase was the same for the three tasks and included 25 two alternative forced-choice trials (2AFC) in which participants had to choose between two triplets (separated by 500ms). On each trial, participants heard a real triplet (that had appeared in the exposure stream) either followed or preceded by a foil triplet (The order was counterbalanced so that on half of the trials the real triplets appeared first). Foil triplets were constructed by taking the first syllable/sound/drawing from one triplet, followed by the second syllable/sound/drawing from another triplet, and the third syllable/sound/drawing from a third triplet. Thus, each element in the foil triplets appeared in a similar position in real triplets, but with different surrounding syllables (for example, "kilome" or "dubega"). This created a difference in the statistical properties of the real triplets and the foils: while the TPs between every two adjacent elements within a real triplet are 1, the TPs between every two syllables in a foil test item are 0 , as participants never heard these elements one after the other during familiarization. If participants learn the statistical properties of the stream, they should be able to distinguish between real triplets and foils. Scores on each task could range from $0 \%$ accuracy $(0 / 25$ trials correct) to $100 \%$ (25/25 trials correct). Trials were presented in random order, with the constraint that the same triplet/foil did not appear in two consecutive trials 


\section{Study 1: The reliability of child-friendly SL tasks in adults Participants}

52 adults participated in both testing sessions (mean age 23 years, 38 females and 19 males). All were university students and received payment for participation. All were native Hebrew speakers and none had any learning, hearing or language impairments.

\section{Procedure}

Participants were tested in a quiet room in the lab while seated in front of a computer. They completed all four tasks (three SL tasks and working memory tasks) in both sessions. Task order was semi-randomized. There were three possible orders for the SL tasks (linguisticauditory, visual, non-linguistic auditory; visual, non-linguistic auditory, linguistic auditory; non-linguistic auditory, linguistic auditory, visual). Verbal memory was tested in between the SL tasks. Participants were tested in different orders in each session. Each session took thirty minutes to complete. In the two auditory tasks, participants were told they will be learning a novel alien language (linguistic auditory) or song (non-linguistic auditory). Following exposure, participants were asked to say which of two words/ sound sequences was more like the language/song they just heard. In the visual task, they were told that they were about to see objects that aliens are taking back to their country. Following exposure, participants were asked to help the spaceship commander remember which objects were taken into the spaceship together. After hearing/seeing both possibilities, participants were asked to press either " 1 " or " 2 " according to whether they thought the correct triplet was the first or the second they heard. In cases where participants felt they didn't know the answer, they were encouraged to try and guess. 


\section{Results}

Performance on SL tasks in the two sessions. Task order did not have a significant effect on performance in any of the SL tasks in both sessions, so results from the three orders were collapsed ( $p$ 's $>0.4$ for all tasks). Gender also did not affect performance ( $p$ 's $>0.3$ for all tasks). Adults showed learning in all tasks (see Table 1). Performance did not improve significantly for any of the tasks ( $p$ 's $>0.2$ ), as has been previously found (Siegelman \& Frost, 2015). Performance was better on the visual task compared to the two auditory ones (visual vs. linguistic auditory: session $1 \mathrm{t}(51)=5.21, p<.001$, session $2 \mathrm{t}(51)=4.19, \mathrm{p}<.001$; visual vs. non-linguistic auditory: session $1 \mathrm{t}(51)=6.77, p<.001$, session $2 \mathrm{t}(51)=6.4, p<.001)$, in line with previous findings with (Siegelman \& Frost, 2015). The ranges and SDs for all tasks were similar in both sessions, indicating there wasn't a change in the distribution of performance.

Table 1: Means and ranges for all tasks in both sessions for adults (including SD in brackets and comparison to chance level)

\begin{tabular}{|l|c|c|c|c|}
\hline Task & $\begin{array}{c}\text { First Session } \\
\text { Accuracy }\end{array}$ & Range & $\begin{array}{c}\text { Second Session } \\
\text { Accuracy }\end{array}$ & \\
\hline Linguistic ASL & $71 \%(0.13)^{* * *}$ & $40 \%-100 \%$ & $74 \%(0.14)^{* * *}$ & $40 \%-100 \%$ \\
\hline Non-linguistic ASL & $71 \%(0.15)^{* * *}$ & $44 \%-100 \%$ & $69 \%(0.19)^{* * *}$ & $24 \%-100 \%$ \\
\hline Visual SL & $87 \%(0.15)^{* * *}$ & $44 \%-100 \%$ & $88 \%(0.18)^{* * *}$ & $32 \%-100 \%$ \\
\hline Working memory & $10.2(1.78)$ & $6-14$ & $10.4(1.54)$ & $8-13$ \\
\hline
\end{tabular}

Assessing the reliability of the SL tasks We evaluate the reliability of the SL by looking at the internal reliability and consistency of tasks within each session and by examining their test retest reliability across the two sessions. To examine the internal reliability and consistency, we calculated the split-half and Cronbach's alpha coefficients for each task in each session (using 


\section{Under review}

the alpha function in R, Revelle, 2015): both measures give an indication of how well each item predicts overall performance, and whether performance on different items is correlated, as would be expected if knowledge of the triplets reflects learning of the statistical structure of the input. To evaluate the test re-test reliability of the SL tasks we looked at the correlation in performance on the same task in the two sessions. Table 2 shows the three reliability measures for all tasks in both sessions for adults.

Table 2: Internal consistency and reliability measures for all tasks in both sessions (with 95\%

CIs in brackets).

\begin{tabular}{|c|c|c|c|c|c|c|}
\hline & \multicolumn{2}{|c|}{ Linguistic Auditory } & \multicolumn{2}{|c|}{ Non-linguistic Auditory } & \multicolumn{2}{|l|}{ Visual } \\
\hline Internal & 0.65 & 0.68 & 0.69 & 0.72 & 0.73 & 0.75 \\
\hline consistency & {$[0.58,0.71]$} & {$[0.61,0.75]$} & {$[0.61,0.77]$} & {$\left[\begin{array}{ll}0.61 & 0.82\end{array}\right]$} & {$[0.63,0.81]$} & {$[0.66,0.85]$} \\
\hline Split-half & 0.72 & 0.78 & 0.78 & 0.82 & 0.87 & 0.91 \\
\hline reliability & {$[0.58,0.82]$} & {$[0.65,0.87]$} & {$[0.66,0.86]$} & {$[0.71,0.89]$} & {$[0.80,0.92]$} & {$[0.85,0.95]$} \\
\hline Test re-test & \multicolumn{2}{|c|}{$\mathrm{r}=0.61 * * *[.41, .76]$} & \multicolumn{2}{|c|}{$\mathrm{r}=0.70^{* * *}[.53,0.82]$} & \multicolumn{2}{|c|}{$\mathrm{r}=0.45^{* * *}[.20,0.64]$} \\
\hline
\end{tabular}

Importantly, all three tasks showed moderate reliability that was generally lower than standard psychometric norms. The Cronbach alpha coefficients ranged between $0.65-0.75$, which is lower than the advised values of 0.8-0.95 (see Steiner, 2003). While all three testretest correlations were significant, their values were lower what is expected from tools assessing individual traits (Ellis, 2013; Nunnally \& Bernstein, 1994). Unsurprisingly, the estimates are somewhat lower than found for similar SL tasks that were modified to better capture individual differences by increasing the test trials and varying their difficulty and kind (Siegelman, Bogaerts \& Frost, 2017). This study shows that our measures show moderate reliability in adults while highlighting their limitation as a tool for assessing individual differences, even in adults. 


\section{Under review}

Correlations between the tasks Table 3 shows the correlation between the three SL tasks in the two sessions. The pattern of correlations was stable across sessions, another indication of task stability: the two auditory tasks were not correlated, while the non-linguistic auditory task and the visual one were. While this pattern of correlation seems counter-intuitive because the tasks do not group together based on modality, it is compatible with a set of recent findings suggesting that tasks using linguistic stimuli (like syllables) behave differently from tasks using non-linguistic stimuli, regardless of modality (Siegelman et al. under review; Shufaiyna \& Arnon, under review). An additional stable pattern was the positive correlation between the SL tasks and working memory, a pattern that has been reported in some adult studies (Misyak \& Christiansen, 2012) but not others (Siegelman \& Frost, 2015). We return to both findings in the general discussion.

Table 3: Simple Bivariate Pearson correlations between the different SL tasks in both sessions, with $95 \%$ CIs in brackets

\begin{tabular}{lllllll}
\hline & \multicolumn{5}{c}{ First Session } & \multicolumn{5}{c}{ Second Session } \\
& Non-linguistic & Visual & WM & Non-linguistic & Visual & WM \\
Linguistic & 0.07 & -0.09 & $\mathbf{0 . 2 8 *}$ & 0.20 & -.09 & 0.23 \\
Non-linguistic & & $\mathbf{0 . 4 1 * *}$ & $0.32 *$ & & $\mathbf{0 . 3 9 * *}$ & $\mathbf{0 . 2 6 *}$ \\
Visual & & $\mathbf{0 . 3 8 * *}$ & & $\mathbf{0 . 3 1 *}$ \\
$* \mathrm{p}^{*}<.05,{ }^{* *} \mathrm{p}<0.01$ & & & & & &
\end{tabular}

\section{Study 2: Reliability of SL measures in children}

\section{Participants}

44 children participated in the first testing session. Three children were absent from school during the second testing session, so the final sample comprised 41 children (19 boys). 


\section{Under review}

Children were in second or third grade (mean age $8 ; 2$, range $7 ; 2-9 ; 0$ ); all were native Hebrew speakers and none had known learning, hearing or language impairments. Parental consent was obtained for all participating children. Children received a small educational reward.

\section{Procedure}

Children were tested in a quiet room in their school. The procedure was identical to that of adults with the only difference being that an experimenter sat next to the child and read out the instructions to them. Children completed all four tasks (three SL tasks and working memory tasks) in both sessions in the three possible orders. Verbal memory was tested in between the SL tasks. Children were tested in different orders in each session. Each session took thirty minutes to complete. At the start of each session, children were told they will be playing a few games with the experimenter and can stop at any time. During the linguistic auditory task, children were told that they were about to hear an alien language, and were then exposed to the familiarization stream using sound-cancelling headphones. Following exposure, children were told that they were about to hear an alien who is not a good speaker of the alien language, and that they must help him by telling him which of the two words he says sounds more like the alien language they just heard. In the non-linguistic auditory task, children were told to learn an alien song and then say which sound sequence is more like the alien song they just heard. In the visual task, children were told that they were about to see objects that aliens are taking back to their country. Following exposure, children were asked to help the spaceship commander remember which objects were taken into the spaceship together. After hearing/seeing both possibilities, children were asked to press either "1" or "2" according to whether they thought the correct triplet was the first or the second they heard. In cases where children felt they didn't know the answer, the experimenter encouraged them to try and guess. 


\section{Results}

Performance on SL tasks in the two sessions. Table 4 shows group-level performance on all tasks. Task order did not have a significant effect on performance in any of the SL tasks in both sessions, so results from the three orders were collapsed ( $p$ 's $>0.2)$. Gender also did not affect performance ( $p$ 's $>0.6$ ). Children showed learning across modalities and stimulustypes: they were significantly above chance for all tasks in the first session and for the linguistic-auditory and visual task in the second session (see Table 4, first session: visual: $\mathrm{t}(40)=7.48, p<.001$; non-linguistic auditory: $\mathrm{t}(40)=4.43, p<.001$; linguistic auditory: $\mathrm{t}(40)$ $=4.71, p<.001$; second session: visual: $\mathrm{t}(40)=7.24, p<.001$; non-linguistic auditory: $\mathrm{t}(40)$ $=1.44, p=0.15$; linguistic auditory: $\mathrm{t}(40)=7.33, \mathrm{p}<.001)$. Performance improved significantly between sessions only for the linguistic-auditory task $(\mathrm{t}(40)=2.74, p<.01)$. The ranges and SDs for all tasks were similar in both sessions, indicating there wasn't a change in the distribution of performance. Children's performance was lower than that of adults on all three tasks.

Table 4: Means and ranges for all tasks (including comparison to chance performance) in both sessions

\begin{tabular}{|l|c|c|c|c|}
\hline Task & First Session & Range & Second Session & Range \\
& Accuracy & & Accuracy & \\
\hline Linguistic ASL & $57 \%(0.10)^{* *}$ & $40 \%-84 \%$ & $63 \%(0.10)^{* * *}$ & $40 \%-84 \%$ \\
\hline Non-linguistic ASL & $59 \%(0.13)^{* *}$ & $36 \%-92 \%$ & $52 \%(0.11)$ & $32 \%-80 \%$ \\
\hline Visual SL & $69 \%(0.15)^{* * * *}$ & $36 \%-92 \%$ & $69 \%(0.16)^{* * *}$ & $32 \%-96 \%$ \\
\hline Working memory & $6.5(1.39)$ & $4-11$ & $7.1(1.13)$ & $5-10$ \\
$*$ ** Significantly above chance, $\mathrm{p}<.01, * * *$ Significantly above chance, $\mathrm{p}<.001$ & & \\
\hline
\end{tabular}




\section{Under review}

In line with prior findings with children at this age (Raviv \& Arnon, 2017), the visual task showed higher accuracy than the two auditory tasks in both sessions (session one: visual vs. linguistic: $t(40)=3.53, p<.001$, visual vs. non-linguistic: $t(40)=3.20, p<.01$; session two: visual vs. linguistic auditory, $\mathrm{t}(40)=2.26, p<.05$; visual vs. non-linguistic auditory: $\mathrm{t}(40)=$ $6.35, p<.001)$. The relation between the linguistic and non-linguistic auditory tasks changed between sessions: in the first session performance did not differ on the two tasks $((\mathrm{t}(40)=-$ $0.71, p>0.4)$ while in the second session performance was better on the linguistic task compared to the non-linguistic one $(\mathrm{t}(40)=3.85, p<.001)$. The results of the two sessions show that children showed learning; that they were better in the visual task than the auditory one; and that they didn't perform consistently better on one of the auditory tasks.

Assessing the reliability of the SL tasks We evaluated the reliability of the SL tasks using the same reliability measures used with adults (Cronbach alpha coefficients and split-half reliability within session and test re-test reliability across sessions). Table 5 shows the three reliability measures for all tasks in both sessions.

Table 5: Internal consistency and reliability measures for all tasks in both sessions (with 95\%

CIs in brackets).

\begin{tabular}{|c|c|c|c|c|c|c|}
\hline & Linguistic $A$ & ditory & Non-linguist & Auditory & Visual & \\
\hline $\begin{array}{l}\text { Internal } \\
\text { consistency }\end{array}$ & $\begin{array}{l}0.04 \\
{[0.01,0.07]}\end{array}$ & $\begin{array}{l}0.27 \\
{[0.24,0.30]}\end{array}$ & $\begin{array}{l}0.43 \\
{[0.39,0.43]}\end{array}$ & $\begin{array}{l}0.2 \\
{\left[\begin{array}{ll}0.17 & 0.23\end{array}\right]}\end{array}$ & $\begin{array}{l}0.68 \\
{[0.63,0.73]}\end{array}$ & $\begin{array}{l}0.72 \\
{[0.67,0.77]}\end{array}$ \\
\hline $\begin{array}{l}\text { Split-half } \\
\text { reliability }\end{array}$ & $\begin{array}{l}-0.04 \\
{[-0.34,0.26}\end{array}$ & $\begin{array}{l}0.22 \\
{[-.08,0.5]}\end{array}$ & $\begin{array}{l}0.37 \\
{[0.07,0.60]}\end{array}$ & $\begin{array}{l}0.08 \\
{[-0.22,0.38]}\end{array}$ & $\begin{array}{l}0.59 \\
{[0.36,0.76]}\end{array}$ & $\begin{array}{l}0.46 \\
{[0.18,0.67]}\end{array}$ \\
\hline $\begin{array}{l}\text { Test-rest } \\
\text { reliability }\end{array}$ & \multicolumn{2}{|c|}{$\mathrm{r}=0.33, p=.035[0.02,0.57]$} & \multicolumn{2}{|c|}{$\mathrm{r}=0.24, \mathrm{p}=0.1[.06,0.51]$} & \multicolumn{2}{|c|}{$\mathrm{r}=0.01, \mathrm{p}>0.9[-.29,0.31]$} \\
\hline
\end{tabular}




\section{Under review}

There are two important patterns to notice. First, in contrast with the adult data, all reliability measures were well below psychometric standards. Both the Cronbach alpha coefficients and split half-reliability varied greatly between tasks (from 0.04 for the linguistic auditory task to 0.68 for the visual one), and were below the recommended values for standard psychometric tests (Steiner, 2003). All three tasks showed test-rest reliability well below psychometric norms, and well below what we found for adults on the same tasks. Only the linguistic auditory task showed any correlation between sessions, with a test re-test reliability of 0.33 , meaning that only $32 \%$ of the variance is shared between test and re-test. This was also the only task in which triplets were repeated across the two sessions, which should have boosted the correlation. Crucially, working memory did show the expected test re-test reliability between the two sessions $(\mathrm{r}=0.67, p<.001)$, which was similar in magnitude to that reported in the literature for this age group (Gathercole et al. 1994). The second striking pattern is the lack of consistency on the three measures. The auditory-linguistic task, which showed the highest test re-test reliability, had the lowest values on the two other measures. The visual task, which showed the highest internal consistency, had no test retest reliability.

To further explore these findings, we calculated an aggregated SL score for each participant based on the average performance on the three tasks. Taking an aggregated measure may increase the stability of the measure. When using this aggregated measure, the correlation between sessions was still low $(\mathrm{r}=0.31, p=0.046)$, and in fact lower than the correlation between the two linguistic auditory tasks, indicating that the other two tasks were not contributing much to the correlation. Moreover, the empirical and theoretical motivation for using an aggregate score is weak given that performance on the different tasks was not systematically correlated within an individual (see Table 6 below). Second, we asked how many children were individually above chance on each of the tasks. Even when children as a group are above chance, many individual learners may be performing at chance level, making 


\section{Under review}

it hard to interpret any correlation between their scores. Following (Siegelman, Bogaerts, \& Frost, 2017), we used the binomial distribution to determine chance level for an individual learner. Since there were 25 test trials in each task, the threshold was set to 17 or more correct responses $(p<.05)$. Very few individuals who were above chance in the same task in both sessions (another indication of low reliability): only 5 children for the linguistic auditory task, 1 for the non-linguistic auditory task, and 14 for the visual one. The examination of individual patterns highlights another problematic aspect of these tasks (which has been previously noted by Siegelman et al. 2017a): the relatively low performance rates mean that many participants are at chance, making their scores uninformative for predicting variation in other measures. We return to this point in the discussion.

Correlations between the tasks Table 6 shows the correlation between the three SL tasks in the two sessions. In contrast with the adult findings, and in line with their low reliability, the pattern of correlations between SL tasks was not stable across the two sessions (see Table 3). In the first session, the two auditory tasks were correlated, but neither correlated with the visual task. However, an opposite pattern was observed in the second session: now the two auditory tasks were correlated with the visual one but not with each other. The lack of stable correlation pattern makes it hard to interpret the relation between the three tasks.

Table 6: Simple Bivariate Pearson correlations between the different SL tasks in both sessions, with $95 \%$ CIs in brackets

\begin{tabular}{lllllll}
\hline & \multicolumn{5}{c}{ First Session } & \multicolumn{5}{c}{ Second Session } \\
& Non-linguistic & Visual & WM & Non-linguistic & Visual & WM \\
Linguistic & $\mathbf{0 . 3 8 *}$ & -0.14 & 0.04 & -0.20 & 0.28 & 0.07 \\
Non-linguistic & & 0.12 & -0.11 & & $\mathbf{0 . 3 3 *}$ & 0.03 \\
Visual & & -.014 & & & -.007 \\
*Significantly above chance, $\mathrm{p}<.05$ & & & & & &
\end{tabular}




\section{Under review}

The relation between the SL tasks and working memory was stable across sessions, and different from what was found with adults: none of the SL tasks were correlated with verbal working memory in both sessions, replicating previous findings with children (Kidd, 2012; Kidd \& Arciuli, 2016). Importantly, the lack of reliability makes it hard to interpret any of these correlations in a meaningful way.

\section{Discussion}

We set out to assess the reliability of three SL tasks modeled on ones previously used in the child individual difference literature. While there is growing use of such tasks to predict individual differences in language outcomes during development, no study to date has shown that they measure a stable property within a child. This is important in light of previous reports that such tasks have psychometric shortcomings as measures of individual differences even in adults (Siegelman et al. 2017ab), and the possibility that these shortcomings are more pronounced in children. To examine this, we tested children and adults on two auditory SL tasks and a visual one two months apart. We also assessed working memory in both sessions to make sure that it shows the expected reliability. We looked at two types of reliability: within session (assessed using split-half and Cronbach alpha's coefficients) and between sessions (using test retest reliability).

The first study showed that our three SL tasks had moderate reliability in adults. These findings replicate previous investigations of task reliability in adult learners in two ways: by indicating that SL measures developed to assess group-level performance can capture stable variation in adults (Potter, Wang \& Saffran, 2017; Siegelman \& Frost, 2015) and by illustrating their shortcomings: reliability was lower than psychometric norms and lower than found when using SL tasks modified to increase their psychometric validity (Siegelmen, Bogaerts \& Frost, 2017). The second study looked at the reliability of the same tasks in 


\section{Under review}

children and found a strikingly different pattern: while children showed learning as a group on all tasks, all reliability measures were well below accepted norms, raising concern about their use as measures of individual differences in development. There was no correlation in performance between sessions for the visual task and the non-linguistic auditory one. The correlation for the auditory linguistic task - which is based on Saffran et al. 1996 and has been used in numerous papers since - was very low $(\mathrm{r}=0.3)$. This was also the only task where learners were exposed to same triplets in both sessions, a factor which may have inflated the correlation. Even under these conditions, the correlation had a value well below psychometric standards (Ellis, 2013). The lack of stability was also reflected in the relation between the different tasks. Unlike the adult data, we found no stable correlations between the different SL tasks: in the first session, the two auditory tasks were correlated with each other (but not with the visual task), suggesting a modality-based division. In the second session, however, the only correlation was across modality (between the non-linguistic and the visual task).

In contrast, verbal working memory showed the expected high reliability across sessions (Gathercole et al. 1994), suggesting that the lack of reliability on the SL tasks is not related to our sample, but reflects something more meaningful about the SL tasks themselves. To give a comparison, the test-retest reliability of the PPVT, a commonly used vocabulary measure, is between 0.91-0.94 with a split half of 0.86-0.97 and Cronbach alpha's of 0.920.98 (Dunn \& Dunn, 1997). The test re-test reliability of the digit span (the verbal working memory task we used) was 0.68 in adults, with a Cronbach's alpha of 0.83 (Waters \& Caplan, 2003). The low reliability we found is consistent with several patterns in the existing developmental data. The correlations tend to be low (ranging between 0.1 and 0.32 , see (Siegelman, Bogaerts, Christiansen, et al., 2017) and vary quite a bit even when using the same language measures. For instance, Kidd (2012) and Kidd \& Arciuli (2016) found no correlation between SL and vocabulary measures in children while Spencer et al (2015) did 


\section{Under review}

(though small ones). Importantly, none of the previous child individual difference studies assessed the reliability of the tasks used.

Taken together these results indicate that the SL tasks we examined cannot be used as a reliable measure of individual differences in children. Since these tasks share important psychometric properties with ones previously used in the developmental literature, they raise a more general concern about existing findings on the relation between SL and language outcomes. Low reliability could lead both to the detection of correlations that are not really there (spurious correlations) and to underestimating true correlations (due to measurement error), making it hard to draw strong conclusions from existing reports of correlations between SL measures and linguistic outcomes. The same problem holds for two additional theoretical questions: the domain-generality of SL in development and its' relation to other cognitive skills: the lack of reliability argues caution in interpreting correlations with other measures or other SL tasks. To give an example, based on the results of the first session alone, we could argue for a modality-sensitive characterization of SL: individual performance was correlated within modality but not between modality. The second session, however, gave rise to a different pattern, not compatible with this conclusion. Importantly, our findings do not undermine the basic claim that humans are capable of extracting distributional information from their input, and that this mechanism plays a role in language acquisition. The lack of reliability does not invalidate findings at the group-level: current tasks are informative for studying which relations (and when) children can learn (though their psychometric weakness means that they are prone to noise and measurement error). However, they cast doubt on their appropriateness as a measure for individual differences in development.

Before discussing the source of the low reliability in children, we briefly address the pattern of correlations between the three SL tasks in our adult data. Examining performance on several SL tasks across modalities can shed light on the modality-specific nature of SL. 


\section{Under review}

While SL is found across modalities (e.g., Conway \& Christiansen, 2005), there is evidence for modality-based differences in performance in both children (Raviv \& Arnon, 2017) and adults (Emberson et al., 2011; Frost, Armstrong, Siegelman, \& Christiansen, 2015). From the perspective of individual differences, adults' performance on visual and auditory tasks is not correlated (Erickson, Kaschak, Thiessen \& Berry, 2016; Siegelman \& Frost, 2015), a finding used to argue that SL is not a unitary capacity, but one that is sensitive to modality. Our findings give rise to more complex pattern: In both sessions, performance was not correlated on the two auditory tasks, but was correlated between the visual and the non-linguistic auditory tasks. That is, performance patterned together based on stimuli-type (linguistic vs. non-linguistic) rather than modality (auditory vs. visual). This pattern is in line with a set of recent findings suggesting that the nature of stimuli, and in particular whether it is linguistic or non-linguistic, is an important factor in explaining SL performance. SL tasks using linguistic stimuli (syllables) are more affected than visual tasks by the exact stimuli used and show evidence of L1 influences (Siegelman, Bogaerts, Elazar, Arciuli \& Frost, under review). Developmentally, the effect of age on performance seems different for tasks using linguistic stimuli: while performance on visual and non-linguistic auditory SL improves during childhood (ages 5-12 years), performance on linguistic auditory SL does not (Shufaniya \& Arnon, under review). Since studies finding modality-based differences often use linguistic auditory tasks (Emberson et al. 2011; Siegelman \& Frost, 2015), previously reported differences (and similarities) may be driven not only by modality by also by the specific stimuli use. Our findings offer further evidence for similarities across modalities and point to the linguistic nature of stimuli as a possible cause for modality-based differences.

Returning to the reliability data, where does task instability come from and why is it more pronounced in children? One possibility is that SL itself is not a stable individual property, but rather one that is affected by an unknown combination of other cognitive 


\section{Under review}

abilities, like memory and attention. Children may be more vulnerable to such influences, explaining why reliability was higher in adult learners in this study and in studies using similar tasks (Siegelman, Bogaerts, \& Frost, 2017; Siegelman \& Frost, 2015). However, a more likely interpretation is that SL is a stable property also in children, but one that is not well measured by the tasks we currently use. Indeed, low reliability in children has also been found in other learning tasks that are reliable in adults: a recent paper assessed the reliability of commonly used procedural memory measures (SRT, Hebb learning and contextual cuing), and found that they have very low reliability in children and do not have stable correlations with language or literacy measures (West, Vadillo, Shanks \& Hulme, 2017).

The current study does not allow us to discern which aspects of SL tasks are responsible for their low reliability in children, but points to several plausible directions. The low reliability may be driven by task properties (number of test trials, level of difficulty), its' explicit nature, or a combination of both. The fact that many children did not show learning at an individual level in both sessions (around $80 \%$ in the two auditory tasks and $50 \%$ in the visual one), and that they showed worse performance than adults, indicates that the task was difficult. This is not a unique feature of the three tasks used here: group accuracy rates were similar or higher in this study compared to other ones with children of similar ages (Raviv \& Arnon, 2017; Kidd \& \& Arciuli, 2016; Spencer et al. 2015). These accuracy levels are problematic because they do not generate the wide distribution of performance needed for individual difference studies. While it is clear that tasks need to be made easier, there is not a straightforward solution of how to do so. Reducing the number of triplets may reduce memory demands, but it also impacts the statistical distinction between elements within and between triplets (having only three triplets instead of five means that the TPs within a triplet is 1 while the TPs between triplets is increased to 0.5 instead of 0.25 ). Increasing exposure times may also not solve the problem. Accuracy rates in our study were similar to those found 


\section{Under review}

when exposing children of the same age to a word segmentation task with four times the exposure length of the current study (59\% in Saffran et al. 1997 vs. our 57\%). In fact, a recent study using online measures to track the trajectory of visual statistical learning in adults showed that learning is already evident after seven repetitions of each triplet and does not increase in magnitude with increased exposure (Siegelman, Bogaerts, Kronenfeld \& Frost, 2017). That is, it is unclear whether increasing exposure length will improve learning significantly.

While task difficulty needs to be addressed, several findings suggest that it is not the major factor driving the low reliability in children. First, test re-test reliability was not higher for the visual task, which had higher accuracy overall and where performance was similar to that of adults (Raviv \& Arnon, 2017). Second, the proportion of individual children showing above chance performance was similar to that found in adults (Siegelman, Bogaerts \& Frost, 2017), where task reliability is higher. One likely possibility is that the children are more affected than adults by the explicit nature of the task and that reliability will be improved by the development of more implicit measures of learning. Such measures have been used with adults (self-paced visual presentation; Karuza, Farmer, Fine, Smith, \& Jaeger, 2014; Rapid Serial Auditory Presentation; Franco, Eberlen, Destrebecqz, Cleeremans, \& Bertels, 2015), and do show both reliability and stable correlations with offline performance (Siegelman, Bogaerts, Kronenfeld \& Frost, 2017). Given similar debates on the difference between online and offline measures of behavior in children (for example in the theory of mind literature), this seems like the more promising avenue for addressing the low reliability,. We are currently developing online measures that can be used to assess both auditory and visual SL over a range of ages, from infancy to adulthood.

Interestingly, even the move to more implicit methods may not be enough. A similar debate about the ability of SL measures to predict individual variation has taken place within 


\section{Under review}

the literature on infant speech (see Cristia, Seidl, Junge, Soderstrom, \& Hagoort, 2014). The logic is similar to that used in the SL literature: being better at detecting linguistic regularities (phonemes in this case) should lead to better (or faster) language learning outcomes. This claim is supported by findings showing positive correlations between speech perception measures and vocabulary size (e.g., Tsao, Liu, \& Kuhl, 2004). However, as in the case of the SL literature, there is little data on the stability and reliability of speech perception measures in infancy. In a recent paper, (Cristia et al., 2014) offer a theoretical and methodological critique of these findings highlighting the psychometric weakness of these speech perception measures, which like SL tasks, were developed to assess group-level learning and not individual differences: "Making the jump from correlation to causation requires multidisciplinary approaches and an improvement of the measurements used" (Crista et al. 2014, p, 2). Similar critiques are offered in a recent study questioning the reliability of sequence learning in adults and its' ability to serve as a stable measure of individual differences (Bogaerts, Siegelman, Ben-Porat, \& Frost, 2017).

These debates highlight the similar challenges facing the use of SL as a predictor of individual differences in language learning. Here also, there is need to systematically investigate the stability and strength of SL as a predictor and to develop additional tasks which assess learning implicitly and are better suited to assess individual differences. Until that is done, little can be concluded about the relation between variation in SL and individual differences in language learning outcomes.

Acknowledgements We wish to thank Noam Siegelman for comments and help with the statistical analyses, Louisa Bogaerts and Ram Frost for comments and helpful discussions. We thank Zohar Aizenbud and Amir Efrati for assistance in programming the experiments and coordinating the testing. We also want to thank the research assistants who collected the 


\section{Under review}

data: Yuval Braeman, Noa Bar, Shira Zicherman, Hilla Merhav, Amir Efrati, Amir Shufaniya, and Hana Gerchikov. We thank the children, parents and teachers at the Givat Mesu'aa primary school. The research was funded by an Israeli Science Foundation grant to the first author (grant number 584/16). 


\section{Under review}

Appendix A: list of stimuli used in the three SL tasks

Linguistic auditory task (syllables): du, ka, me, ge, di, no, ki, mu, ga, na, lo, bi, to, be, lo

Non-linguistic auditory task (familiar sounds): "bird tweet", "running water", "goat bleat", "opening door", "dog bark", "bouncing ball", "trumpet", "cat meow", "duck quack", "frog quack", "cuckoo clock", "bell", "chord", "whistle" and "cow moo".

Visual task: black-and-white drawings (taken from Alario \& Fernand, 1999) of house, book, cake, dog, cat, airplane, shoe, fish, ball, banana, fork, flower, bottle, chair and butterfly.

Example images:

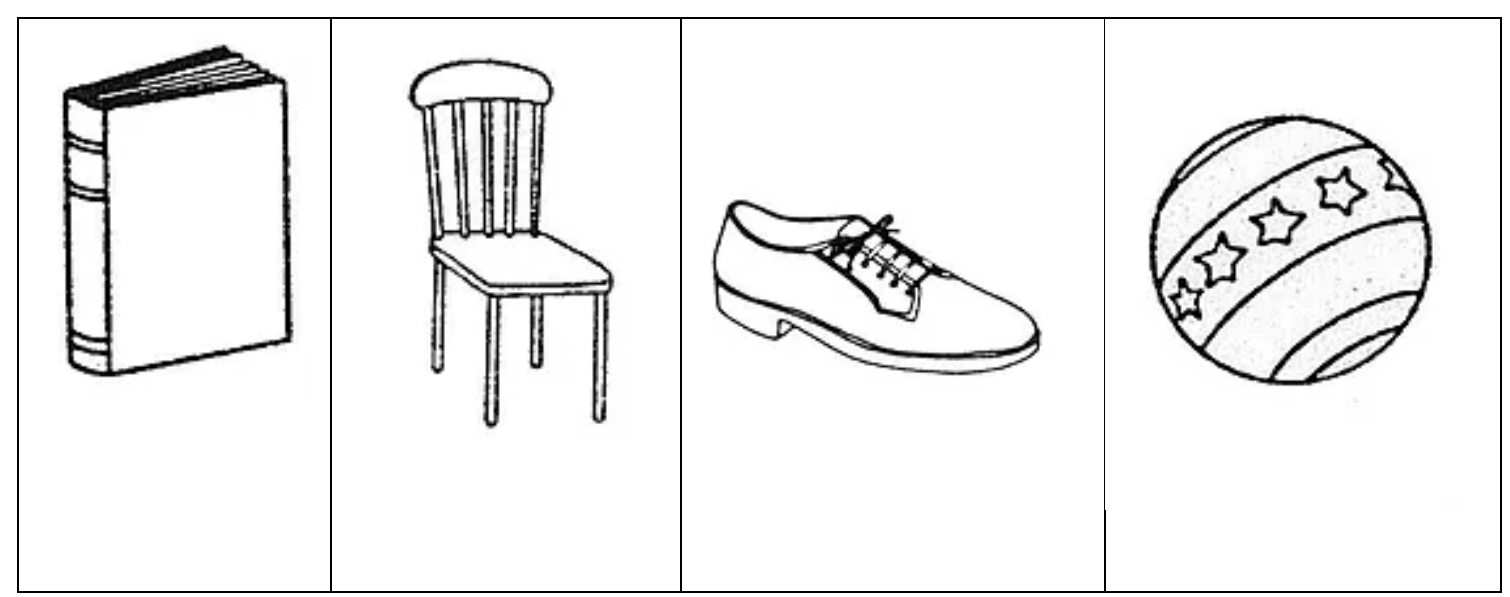




\section{References}

Alario, F. X., \& Ferrand, L. (1999). A set of 400 pictures standardized for French: norms for name agreement, image agreement, familiarity, visual complexity, image variability, and age of acquisition. Behavior Research Methods, Instruments, \& Computers, 31(3), 531552. http://doi.org/10.3758/BF03200732

Arciuli, J., \& Simpson, I. C. (2011). Statistical learning in typically developing children: The role of age and speed of stimulus presentation. Developmental Science, 14(3), 464-473. http://doi.org/10.1111/j.1467-7687.2009.00937.x

Arciuli, J., \& Simpson, I. C. (2012). Statistical Learning Is Related to Reading Ability in Children and Adults. Cognitive Science, 36(2), 286-304.

Bogaerts, L., Siegelman, N., Ben-Porat, T., \& Frost, R. (2017). Is the Hebb repetition task a reliable measure of individual differences in sequence learning? The Quarterly Journal of Experimental Psychology, O(0), 1-35. http://doi.org/10.1080/17470218.2017.1307432

Bulf, H., Johnson, S. P., \& Valenza, E. (2011). Visual statistical learning in the newborn infant. Cognition, 121(1), 127-132. http://doi.org/10.1016/j.cognition.2011.06.010

Conway, C. M., Bauernschmidt, A., Huang, S. S., \& Pisoni, D. B. (2010). Implicit statistical learning in language processing: word predictability is the key. Cognition, 114(3), 35671. http://doi.org/10.1016/j.cognition.2009.10.009

Conway, C. M., \& Christiansen, M. H. (2005). Modality-constrained statistical learning of tactile, visual, and auditory sequences. Journal of Experimental Psychology. Learning, Memory, and Cognition, 31(1), 24-39. http://doi.org/10.1037/0278-7393.31.1.24

Cristia, A., Seidl, A., Junge, C., Soderstrom, M., \& Hagoort, P. (2014). Predicting individual variation in language from infant speech perception measures. Child Development, 85(4), 1330-1345. http://doi.org/10.1111/cdev.12193

Dunn, L. M. \& Dunn, L. M. (1997). PPVT-III : Peabody picture vocabulary test. Circle Pines, 
Under review

$\mathrm{MN}$ :American Guidance Service

Ellis, E. M., Robeldo Gonzales M., D. G. O. (2013). Previously Published Works. Language Learning and Development, 1, 1-15. http://doi.org/10.1016/j.virol.2015.08.001

Erickson, L. C., \& Thiessen, E. D. (2015). Statistical learning of language: Theory, validity, and predictions of a statistical learning account of language acquisition. Developmental Review, 37, 66-108. http://doi.org/10.1016/j.dr.2015.05.002

Frost, R., Armstrong, B. C., Siegelman, N., \& Christiansen, M. H. (2015). Domain generality versus modality specificity: the paradox of statistical learning. Trends in Cognitive Sciences, 1-9. http://doi.org/10.1016/j.tics.2014.12.010

Frost, R., Siegelman, N., Narkiss, a., \& Afek, L. (2013). What Predicts Successful Literacy Acquisition in a Second Language? Psychological Science, 24(7), 1243-1252. http://doi.org/10.1177/0956797612472207

Gathercole, S. E., Willis, C. S., Baddeley, A. D., \& Emslie, H. (1994). The children's test of nonword repetition: A test of phonological working memory. Memory, 2(2), 103-127. http://doi.org/10.1080/09658219408258940

Havron, N., \& Arnon, I. (2017). Minding the gaps: literacy enhances lexical segmentation in children learning to read*. Journal of Child Language, 1-23. http://doi.org/10.1017/S0305000916000623

Isbilen, E. S., Mccauley, S. M., \& Christiansen, M. H. (2017). Testing Statistical Learning Implicitly: A Novel Chunk-based Measure of Statistical Learning. Proceedings of the 39th Annual Conference of the Cognitive Science Society, (May), 564-569.

Kidd, E. (2012). Implicit statistical learning is directly associated with the acquisition of syntax. Developmental Psychology, 48(1), 171-184. http://doi.org/10.1037/a0025405

Kidd, E., \& Arciuli, J. (2016). Individual Differences in Statistical Learning Predict Children's Comprehension of Syntax. Child Development, 87(1), 184-193. 
Under review

http://doi.org/10.1111/cdev.12461

Kirkham, N. Z., Kirkham, N. Z., Slemmer, J. a, Slemmer, J. a, Johnson, S. P., \& Johnson, S. P. (2002). Visual statistical learning in infancy: Evidence for a domain-general learning mechanism, 83, 4-5.

Lany, J., Shoaib, A., Thompson, A., \& Graf Estes, K. (2017). Infant statistical-learning ability is related to real-time language processing. Journal of Child Language, 1-24. http://doi.org/10.1017/S0305000917000253

Mainela-Arnold, E., \& Evans, J. L. (2014). Do statistical segmentation abilities predict lexical-phonological and lexical-semantic abilities in children with and without SLI? Journal of Child Language, 41(2), 327-351. http://doi.org/10.1017/S0305000912000736

Maital, S. L., Dromi, E., Sagi, A., \& Bornstein, M. H. (2000). The Hebrew Communicative Development Inventory: language specific properties and cross-linguistic generalizations. Journal of Child Llnguage, 27(1), 43-67. http://doi.org/10.1017/S0305000999004006

Misyak, J. B., \& Christiansen, M. H. (2012). Statistical Learning and Language: An Individual Differences Study. Language Learning, 62(1), 302-331. http://doi.org/10.1111/j.1467-9922.2010.00626.x

Misyak, J. B., Christiansen, M. H., \& Bruce Tomblin, J. (2010). Sequential expectations: the role of prediction-based learning in language. Topics in Cognitive Science, 2(1), 138-53. http://doi.org/10.1111/j.1756-8765.2009.01072.x

Newman, R., Ratner, N. B., Jusczyk, A. M., Jusczyk, P. W., \& Dow, K. A. (2006). Infants' early ability to segment the conversational speech signal predicts later language development: A retrospective analysis. Developmental Psychology, 42(4), 643-655. http://doi.org/10.1037/0012-1649.42.4.643

Nunnally, J., \& Bernstein, I. (1994). Psychometric theory (3rd ed.). New York: McGraw-Hill Plude, D. J., Enns, J. T. \& Brodeur, D. (1994). The development of selective attention: a life- 
Under review

span review, Acta Psychologia, 86, 227-272.

Potter, C. E., Wang, T., \& Saffran, J. R. (2017). Second Language Experience Facilitates Statistical Learning of Novel Linguistic Materials. Cognitive Science, 41, 913-927.

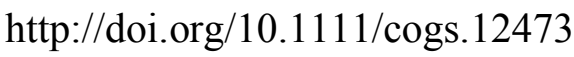

Raviv, L., \& Arnon, I. (2017). The developmental trajectory of children's statistical learning abilities. Developmental Science, 1, 1-13. http://doi.org/10.1111/desc.12593

Revelle, W. (2016). psych: Procedures for Personality and Psychological Research. R Package, 1-358. Retrieved from http://personality-project.org/r/psych-manual.pdf

Romberg, A. R., \& Saffran, J. R. (2010). Statistical learning and language acquisition. WIREs Cogn Sci, 906-914. http://doi.org/10.1002/wcs.78

Saffran, J. R., Aslin, R. N., \& Newport, E. L. (1996). Statistical learning by 8-month-old infants. Science (New York, N.Y.), 274(5294), 1926-1928. http://doi.org/10.1126/science.274.5294.1926

Saffran, J. R., Newport, E. L., Aslin, R. N., Tunick, R. a., \& Barrueco, S. (1997). INCIDENTAL LANGUAGE LEARNING:. Listening (and Learning) out of the Comer of Your Ear. Psychological Science, 8(2), 101-105. http://doi.org/10.1111/j.14679280.1997.tb00690.x

Shafto, C. L., Conway, C. M., Field, S. L., \& Houston, D. M. (2012). Visual Sequence Learning in Infancy: Domain-General and Domain-Specific Associations With Language. Infancy, 17(3), 247-271. http://doi.org/10.1111/j.1532-7078.2011.00085.x

Siegelman, N. Bogaters, L. Kronenfeld, O. \& Frost, R. (2017). Redefining "learning" in statistical learning: what does an online measure reveal about the assimilation of visual regularities? Cognitive Science, 1, 1-36.

Siegelman, N., Bogaerts, L., Christiansen, M. H., \& Frost, R. (2017). Towards a theory of individual differences in statistical learning. Transactions of the Royal Society B, 
Under review

372(1711), 20160059. http://doi.org/10.1098/rstb.2016.0059

Siegelman, N., Bogaerts, L., \& Frost, R. (2017). Measuring individual differences in statistical learning: Current pitfalls and possible solutions. Behavior Research Methods, 49(2), 418-432. http://doi.org/10.3758/s13428-016-0719-z

Siegelman, N., Bogaerts, L., Arciuli, J., Elazar, A. \& Frost, R. (under review). Statistical entrenchment: prior knowledge impacts statistical learning performance

Siegelman, N., \& Frost, R. (2015). Statistical learning as an individual ability: Theoretical perspectives and empirical evidence. Journal of Memory and Language, 81, 105-120. http://doi.org/10.1016/j.jml.2015.02.001

Southgate, V., Senju, A., \& Csibra, G. (2007). Action anticipation through attribution of false belief by 2-year-olds. Psychological Science, 18, 587-592

Streiner, D. L. (2003). Starting at the beginning: an introduction to coefficient alpha and internal consistency. Journal of Personality Assessment, 80, 99-103.

Spencer, M., Kaschak, M. P., Jones, J. L., \& Lonigan, C. J. (2015). Statistical learning is related to early literacy-related skills. Reading and Writing, 28, 467-490. http://doi.org/10.1007/s11145-014-9533-0

Tsao, F., Liu, H., \& Kuhl, P. K. (2004). Speech Perception in Infancy Predicts Language Development in the Second Year of Life : A Longitudinal Study Author ( s ): Feng-Ming Tsao, Huei-Mei Liu and Patricia K . Kuhl Published by: Wiley on behalf of the Society for Research in Child Development. Child Development, 75(4), 1067-1084.

Turk-Browne, N. B., Jungé, J. A., \& Scholl, B. J. (2005). The Automaticity of Visual Statistical Learning. Journal of Experimental Psychology: General, 134(4), 552-564.

West, G., Vadillo, M. A., Shanks, D. R., \& Hulme, C. (2017). The procedural learning deficit hypothesis of language learning disorders: We see some problems. Developmental Science, (January), 1-13. http://doi.org/10.1111/desc.12552 
Under review 